\title{
The value of different diffusion-weighted magnetic resonance techniques in the diagnosis of middle ear cholesteatoma. Is there still an indication for echo-planar diffusion-weighted imaging?
}

\author{
Alina Piekarek ${ }^{1, A, B, C, D, E, F, F}$, Tomasz Zatoński 2,B,D,E, Mateusz Kolator ${ }^{2, B, E}$, Joanna Bladowska ${ }^{1, D, E, F}$, Marek Sąsiadek ${ }^{1, D, E, F}$, \\ Anna Zimny ${ }^{1, A, C, D, E}$
}

'Department of Radiology, Wroclaw Medical University, Wroclaw, Poland

2Department of Otolaryngology, Wroclaw Medical University, Wroclaw, Poland

\section{Abstract}

Purpose: The aim of the study was to analyse the value of 2 different diffusion-weighted imaging (DWI) techniques (echo-planar imaging [EPI] and on-echo-planar imaging [non-EPI]) in the diagnosis of cholesteatoma.

\begin{abstract}
Material and methods: Our material consisted of 32 subjects suspected of cholesteatoma, who underwent magnetic resonance imaging of the temporal bone using both EPI and non-EPI DWI. Two independent readers retrospectively analysed magnetic resonance images. Intra- and interobserver agreements as well sensitivity, specificity, and negative (NPV) and positive (PPV) predictive values of both DWI sequences were assessed.
\end{abstract}

\begin{abstract}
Results: Using non-EPI DWI all cholesteatomas were correctly diagnosed by both readers with no false negative nor inconclusive cases and with only one false positive result. Non-EPI DWI revealed high interobserver agreement $(\kappa=1)$ and high correlation with histopathological results $(r=0.895)$. EPI DWI misdiagnosed $27-31 \%$ of cholesteatomas (false negative results), showing also significantly low interobserver agreement $(\kappa=0.373)$ and low correlation with histopathological results ( $r=0.328$ for reader 1 and $r=0.267$ for reader 2). Non-EPI DWI revealed very high sensitivity (100\%), specificity (83.3\%), NPV (100\%), and PPV (96.3\%) in comparison to EPI DWI, which showed lower sensitivity (69.2\%), specificity (66.6-83.3\%), NPV (33.3-38.4\%), and PPV (90.0-94.7\%).

Conclusions: Non-EPI DWI with high sensitivity, specificity, and interobserver agreement is a very reliable technique in detecting middle ear cholesteatoma regardless of the pre- or postoperative state of the ear, and it should entirely replace EPI DWI in clinical practice.
\end{abstract}

Key words: cholesteatoma, diffusion weighted imaging, EPI DWI, non-EPI DWI.

\section{Introduction}

Cholesteatoma is an inflammatory acquired (98\%) or congenital (2\%) process characterized by collecting an exfoliating keratin inside the middle ear cavity. The incidence of middle ear cholesteatomas is around 3/100,000 in children and 9.2/100,000 in adults with a slight male predilection [1,2]. Typically, cholesteatomas cause osseous erosions along involved structures of the middle ear, and if not treated, it can lead to conductive hearing loss and serious intracranial complications. Due to the potentially erosive and progressive character of middle ear cholesteatomas, the first-choice method of treatment is surgical total excision by canal wall up or wall down tympanomastoidectomy, often with ossicles reconstruction and tympanoplasty [3]. Diagnosis and assessment of the dis-

Correspondence address:

Alina Piekarek, Department of Radiology, Wroclaw Medical University, 213 Borowska St., 50-556 Wroclaw, Poland, e-mail: alina1979@op.pl

Authors' contribution:

A Study design · B Data collection · C Statistical analysis · D Data interpretation · E Manuscript preparation · F Literature search · G Funds collection 
ease extension is based on clinical symptoms, otoscopic findings, and imaging. High-resolution computed tomography (HRCT) allows for recognition of bony destruction, precise localization of the opacity, and assessment of postsurgical or congenital additional temporal bone anomalies. However, it is not capable of differentiation between granulation, fibrous tissue, fluid, and cholesteatoma [4]. Magnetic resonance imaging (MRI) techniques have been introduced to distinguish different pathologies within the middle ear, with particular importance of diffusion-weighted imaging (DWI). There are 2 major types of DWI: using echo-planar imaging (EPI) and nonEPI techniques. Both EPI and non-EPI DWI are specific to cholesteatoma, because of its high keratin content detected as a very high signal similarly to epidermoids [5].

EPI DWI is a fast sequence routinely used in brain imaging. It is characterized by low spatial resolution, geometric distortion, and low signal-to-noise ratio (SNR). It is also prone to susceptibility artifacts at air/bone interfaces, which may cause problems while imaging skull base structures such as the middle ear [5-8]. Non-EPI DWI techniques, such as half-Fourier acquisition single-shot turbo spin-echo (HASTE) DWI, PROPELLER (multishot fast spin-echo periodically rotated overlapping parallel lines with enhanced reconstruction) DWI, BLADE DWI, and multishot DWI turbo spin-echo (MS TSE), require longer scanning times compared to EPI DWI, but they are said to reduce susceptibility artifacts, geometric distortions, and section thickness as well as to increase spatial resolution [7-15].

The purpose of our study was to compare the value of EPI DWI and non-EPI PROPELLER DWI in the diagnosis of the middle ear cholesteatoma regardless of the pre- or postoperative state of the ear, as well as to assess the clinical significance and usefulness of these methods on the basis of an evaluation by 2 independent readers (neuroradiologists) experienced in interpretation of DWI.

\section{Material and methods}

\section{Material}

Our material consisted of 32 patients ( 17 females and 15 males), mean age 31.43 years (range from 5 to 77 years) with clinical suspicion of acquired or congenital cholesteatoma, who underwent MRI of the temporal bone and a subsequent surgery with biopsy. Eighteen patients had a history of previous ear surgery due to cholesteatoma, and 14 subjects did not undergo such procedures before (Table 1).

The study was approved by the local Ethical Committee, and written informed consent was obtained from each patient.

\section{Imaging technique}

All patients underwent MR examination of the temporal bone using a $1.5 \mathrm{~T}$ scanner (GE, SIGNA Hdx with a 16-channel HNS coil). The MR study included the following sequences: axial T1- and T2-weighted images, 3D FIESTA (3-dimensional gradient echo fast imaging employing steady-state acquisition: TE/TR $2 / 5 \mathrm{~ms}$, flip angle $65^{\circ}$, FOV $180 \mathrm{~mm}$, slice thickness 1.0/-0.5) as well as echo-planar diffusion-weighted imaging (EPI DWI) and non-echo-planar PROPELLER DWI (non-EPI PROPELLER DWI). Scanning parameters of EPI DWI were as follows: $\mathrm{TR}=7100, \mathrm{TE}=97.4, b$-value $=1000 \mathrm{~s} / \mathrm{mm}^{2}$, thickness: $5.0 / 1.5 \mathrm{~mm}$, NEX 3, matrix $128 \times 128$, time $=$ $1 \mathrm{~min} 18 \mathrm{~s}$. Scanning parameters of non-EPI PROPELLER DWI were as follows: $\mathrm{TR}=5600, \mathrm{TE}=85.2, b$-value $=$ $1000 \mathrm{~s} / \mathrm{mm}^{2}$, thickness: $4.0 / 0.5 \mathrm{~mm}$, NEX 1.5, matrix $128 \times 128$, time $=4 \mathrm{~min} 51 \mathrm{~s}$.

In complicated postoperative cases additionally $3 \mathrm{D}$ fat-saturated T1-weighted sequences after contrast injection $(0.1 \mathrm{mmol} / \mathrm{kg}$ of gadolinium $)$ were obtained.

\section{Image analysis}

Two independent readers blinded to histopathological results evaluated MR images assessing the signal of middle ear/mastoid pathologies on EPI and non-EPI DWI using the following scoring system: 0 - no hyperintensities on DWI, no restricted diffusion (suggested diagnosis - no cholesteatoma); 1 - intermediate signal on DWI, inconclusive case; 2 - evident hyperintensity on DWI, restricted diffusion (suggested diagnosis - cholesteatoma).

\section{Statistical analysis}

Results of DWI interpretation were correlated with the results of biopsy using Spearman's rank coefficient. Both intra- and interobserver agreements in interpretation of EPI and non-EPI DWI were evaluated using Cohen's $\kappa$ analysis. Sensitivity, specificity, as well as negative and positive predictive values (NPV and PPV, respectively) were assessed. A $p$-value $<0.05$ was defined as statistically significant.

\section{Results}

From 64 evaluated ears, 32 were found to be normal both clinically and on MRI, and thus were not included in the further analysis. Thirty-two ears were operated. In 26/32 $(81.25 \%)$ ears cholesteatoma was diagnosed, while in $6 / 32$ (18.75\%) ears chronic otitis without cholesteatoma was found. Twenty-nine lesions (90.6\%) were measurable, and their diameter ranged from 4 to $25 \mathrm{~mm}$ (Table 1).

The results of EPI and non-EPI DWI scoring by 2 readers are shown in Table 1. In the EPI DWI method both readers used all 3 scores $(0,1,2)$ while in the non-EPI DWI only scores 0 and 2 were used. Based on EPI DWI, both readers estimated 10/32 (31.2\%) of cases with score 1 , meaning inconclusive, while in non-EPI DWI there were no inconclusive cases found. 
Table 1. Demographic data of the patients and the results of assessment of echo-planar (EPI) and non-echo-planar (non-EPI) diffusion-weighted imaging (DWI) by 2 independent readers

\begin{tabular}{|c|c|c|c|c|c|c|c|c|c|}
\hline \multirow{2}{*}{$\begin{array}{l}\text { Patient } \\
\text { No }\end{array}$} & \multirow{2}{*}{$\begin{array}{c}\text { Age } \\
\text { (years) }\end{array}$} & \multirow[t]{2}{*}{ Sex } & \multirow{2}{*}{$\begin{array}{c}\text { Transverse } \\
\text { diameter }(\mathrm{mm})\end{array}$} & \multirow{2}{*}{$\begin{array}{c}\text { Pre-/Postoperative } \\
\text { ear }\end{array}$} & \multicolumn{2}{|c|}{ Reader 1} & \multicolumn{2}{|c|}{ Reader 2} & \multirow{2}{*}{$\begin{array}{l}\text { Histopathological } \\
\text { results }\end{array}$} \\
\hline & & & & & EPI DWI & Non-EPI DWI & EPI DWI & Non-EPI DWI & \\
\hline 1 & 9 & $M$ & - & Post & 0 & 0 & 0 & 0 & Chronic otitis \\
\hline 2 & 71 & $M$ & 14 & Post & 1 & 2 & 2 & 2 & $\mathrm{CHOL}$ \\
\hline 3 & 12 & M & 4 & Post & 0 & 2 & 2 & 2 & $\mathrm{CHOL}$ \\
\hline 4 & 30 & $M$ & 20 & Post & 1 & 2 & 1 & 2 & $\mathrm{CHOL}$ \\
\hline 5 & 28 & $M$ & 16 & Post & 1 & 2 & 2 & 2 & CHOL \\
\hline 6 & 6 & $\mathrm{~F}$ & 10 & Post & 2 & 2 & 2 & 2 & $\mathrm{CHOL}$ \\
\hline 7 & 17 & $\mathrm{~F}$ & 18 & Post & 2 & 2 & 1 & 2 & $\mathrm{CHOL}$ \\
\hline 8 & 12 & $M$ & 25 & Post & 1 & 2 & 2 & 2 & $\mathrm{CHOL}$ \\
\hline 9 & 77 & $\mathrm{~F}$ & 13 & Post & 0 & 2 & 1 & 2 & $\mathrm{CHOL}$ \\
\hline 10 & 55 & $\mathrm{~F}$ & 10 & Post & 0 & 2 & 0 & 2 & $\mathrm{CHOL}$ \\
\hline 11 & 12 & $M$ & 25 & Post & 2 & 2 & 2 & 2 & $\mathrm{CHOL}$ \\
\hline 12 & 10 & $\mathrm{~F}$ & 20 & Post & 1 & 2 & 0 & 2 & $\mathrm{CHOL}$ \\
\hline 13 & 9 & $M$ & 15 & Post & 0 & 2 & 0 & 2 & $\mathrm{CHOL}$ \\
\hline 14 & 40 & $\mathrm{~F}$ & 25 & Post & 0 & 2 & 0 & 2 & $\mathrm{CHOL}$ \\
\hline 15 & 38 & $\mathrm{~F}$ & 20 & Post & 1 & 2 & 1 & 2 & CHOL \\
\hline 16 & 55 & $\mathrm{~F}$ & 10 & Post & 0 & 0 & 1 & 0 & Chronic otitis \\
\hline 17 & 5 & $\mathrm{~F}$ & 10 & Post & 0 & 2 & 0 & 2 & $\mathrm{CHOL}$ \\
\hline 18 & 9 & $\mathrm{~F}$ & 15 & Post & 0 & 2 & 1 & 2 & $\mathrm{CHOL}$ \\
\hline 19 & 29 & $M$ & 15 & Pre & 1 & 2 & 2 & 2 & $\mathrm{CHOL}$ \\
\hline 20 & 34 & $M$ & 4 & Pre & 2 & 2 & 2 & 2 & Chronic otitis \\
\hline 21 & 59 & $\mathrm{~F}$ & 20 & Pre & 1 & 2 & 2 & 2 & $\mathrm{CHOL}$ \\
\hline 22 & 5 & $\mathrm{~F}$ & 25 & Pre & 2 & 2 & 1 & 2 & $\mathrm{CHOL}$ \\
\hline 23 & 40 & $\mathrm{~F}$ & 20 & Pre & 2 & 2 & 2 & 2 & $\mathrm{CHOL}$ \\
\hline 24 & 64 & $\mathrm{~F}$ & 20 & Pre & 2 & 2 & 2 & 2 & $\mathrm{CHOL}$ \\
\hline 25 & 27 & $M$ & 5 & Pre & 2 & 2 & 0 & 2 & $\mathrm{CHOL}$ \\
\hline 26 & 66 & M & - & Pre & 0 & 0 & 0 & 0 & Chronic otitis \\
\hline 27 & 46 & $\mathrm{~F}$ & - & Pre & 0 & 0 & 0 & 0 & Chronic otitis \\
\hline 28 & 48 & $M$ & 10 & Pre & 0 & 0 & 0 & 0 & Chronic otitis \\
\hline 29 & 42 & $M$ & 15 & Pre & 1 & 2 & 1 & 2 & $\mathrm{CHOL}$ \\
\hline 30 & 5 & $\mathrm{~F}$ & 15 & Pre & 1 & 2 & 0 & 2 & $\mathrm{CHOL}$ \\
\hline 31 & 37 & $M$ & 15 & Pre & 0 & 2 & 1 & 2 & $\mathrm{CHOL}$ \\
\hline 32 & 9 & $\mathrm{~F}$ & 20 & Pre & 1 & 2 & 1 & 2 & $\mathrm{CHOL}$ \\
\hline
\end{tabular}

- no evident restricted diffusion, suggesting absence of a cholesteatoma, 1 - inconclusive case, 2 - evident restricted diffusion, suggesting presence of a cholesteatoma, CHOL - cholesteatoma

There was a very high correlation between the results of non-EPI DWI and histological findings ( $r=0.895$, $p<0.001)$ for both readers. In non-EPI DWI all 26 cholesteatomas were correctly diagnosed with score 2 (true positive results), and 5/6 (83.3\%) cases of chronic otitis were correctly scored as 0 . Based on non-EPI DWI only in 1 case the score was 2, indicating that cholesteatoma was given to chronic otitis without cholesteatoma, which was a false positive result. There were no false negative results using the non-EPI DWI method (Table 2).
In the case of EPI DWI there was a very poor correlation with histological findings $(r=0.328, p=0.066$ for reader 1 and $r=0.267, p=0.139$ for reader 2$)$. Reader 1 correctly diagnosed only 7/26 (26.9\%) cholesteatomas (true positive results), in 11/26 (42.3\%) cases of cholesteatoma an inconclusive result of EPI DWI was established, and 8/26 (30.7\%) cholesteatomas were diagnosed as an ear without cholesteatoma (false negative results) (Table 2). Based on EPI DWI, reader 1 correctly diagnosed 5/6 (83.3\%) cases of chronic otitis, while 1 case (16.6\%) of chronic otitis was 
Table 2. Comparison of the results of the assessment of echo-planar (EPI) and non-echo-planar (non-EPI) diffusion-weighted imaging (DWI) by 2 independent readers

\begin{tabular}{|l|c|c|c|c|} 
& $\begin{array}{c}\text { Reader 1 } \\
\text { EPI DWI }\end{array}$ & $\begin{array}{c}\text { Reader 2 } \\
\text { EPI DWI }\end{array}$ & $\begin{array}{c}\text { Reader 1 } \\
\text { Non-EPI DWI }\end{array}$ & $\begin{array}{c}\text { Reader 2 } \\
\text { Non-EPI DWI }\end{array}$ \\
\hline True positive results & $7 / 26(26.9 \%)$ & $10 / 26(38.4 \%)$ & $26 / 26(100 \%)$ & $26 / 26(100 \%)$ \\
\hline True negative results & $5 / 6(83.3 \%)$ & $5 / 6(83.3 \%)$ & $5 / 6(83.3 \%)$ & $5 / 6(83.3 \%)$ \\
\hline False positive results & $1 / 6(16.6 \%)$ & $1 / 6(16.6 \%)$ & $1 / 6(16.6 \%)$ & $1 / 6(16.6)$ \\
\hline False negative results & $19 / 26(73.1 \%)$ & $16 / 26(61.5 \%)$ & $0 / 26(0 \%)$ & $0 / 26(0 \%)$ \\
\hline Sensitivity & $69.2 \%$ & $69.2 \%$ & $100 \%$ & $100 \%$ \\
\hline Specificity & $83.3 \%$ & $66.6 \%$ & $83.33 \%$ & $83.3 \%$ \\
\hline PPV & $94.7 \%$ & $90 \%$ & $96.3 \%$ & $96.3 \%$ \\
\hline NPV & $38.4 \%$ & $33.3 \%$ & $100 \%$ & $100 \%$ \\
\hline Interobserver agreement & \multicolumn{2}{|r|}{$\kappa=0.373$} & & $\kappa=1$ \\
\hline
\end{tabular}

PPV - positive predictive value, NPV - negative predictive value, $\kappa$-Cohen's kappa coefficient

overdiagnosed as cholesteatoma (false positive result). Scoring analysis based on EPI DWI by reader 2 showed very similar results, as follows: $10 / 26$ (38.4\%) correctly diagnosed cholesteatomas (true positive results), 9/26 (34.6\%) inconclusive cases, and 7/26 (26.9\%) misdiagnosed cholesteatomas (false negative results). Chronic otitis without a cholesteatoma was correctly diagnosed in 4/6 (66.6\%) cases, $1 / 6$ cases $(16.6 \%)$ was regarded as inconclusive, and 1 case of chronic otitis (6.6\%) was overdiagnosed as cholesteatoma (false positive result) (Table 2 ).

For both readers the intraobserver analysis showed a very weak agreement between the results of EPI and nonEPI DWI $(\kappa=0.238, p=0.009$ for reader 1 and $\kappa=0.235$, $p=0.027$ for reader 2 ). There was also a very weak interobserver agreement between reader 1 and reader 2 regarding EPI DWI $(\kappa=0.373, p=0.007)$, while there was a complete agreement between 2 readers in the results of non-EPI DWI $(\kappa=1, p<0.000001)$ regardless of the pre- or postoperative status of the evaluated ear.

Overall, non-EPI DWI in both pre- and postoperative ears revealed very high sensitivity $(100 \%)$, specificity (83.3\%), NPV (100\%), and PPV (96.3\%) compared to
EPI DWI, showing significantly lower values of sensitivity (69.2\%), specificity (66.6-83.3\%), NPV (33.3-38.4\%), and PPV (90.0-94.7\%) (Table 2, Figures 1-4).

\section{Discussion}

Diagnosis of cholesteatomas is based on clinical, otoscopic, and imaging evaluation, including HRCT and MRI. Highresolution $\mathrm{CT}$ is a recommended method to localize and define cholesteatoma before surgery, especially in cases with bony erosions of the tegmen tympani, the ossicular chain, and walls of the middle ear/mastoid $[4,16]$. Nevertheless, HRCT demonstrates non-specific opacification in the petrous bone, leading to diagnostic difficulties and making this method insufficient to differentiate cholesteatoma from granulation, fibrous tissue, and fluid $[4,16]$.

MRI has significant value in distinguishing cholesteatomas from other pathological processes involving middle ear structures. Diffusion-weighted imaging is especially important in the imaging of cholesteatomas, which due to their high keratin content show restricted diffusion reflected as a high signal on both echoplanar and non-echo-
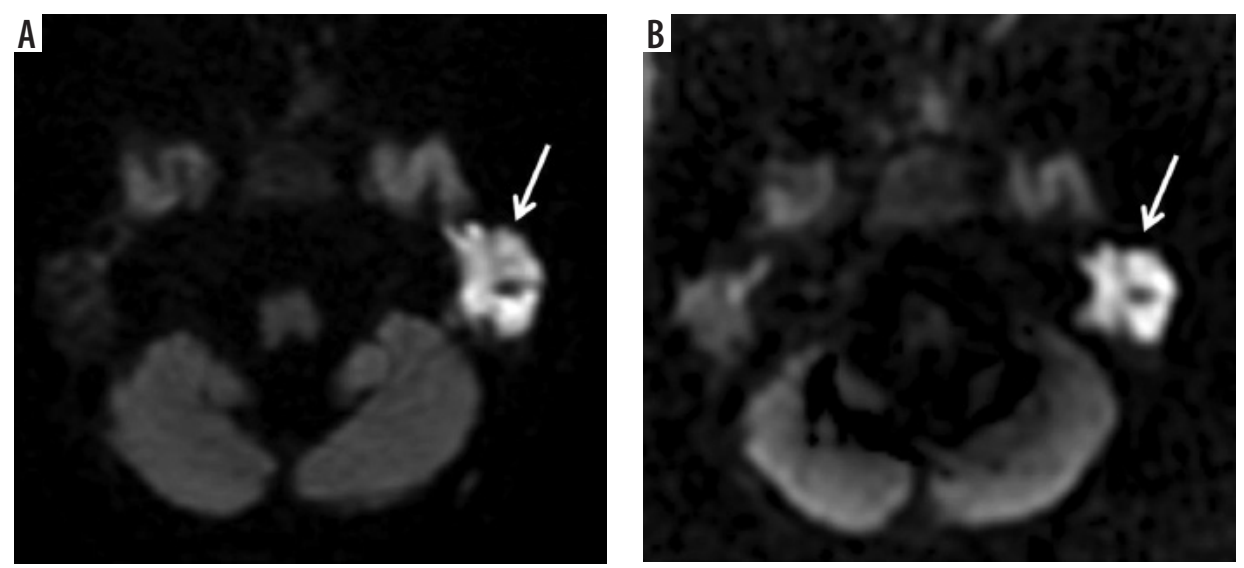

Figure 1. Echo-planar (A) and non-echo-planar (B) diffusion-weighted imaging showing large middle ear cholesteatoma (arrow) with the same image quality 

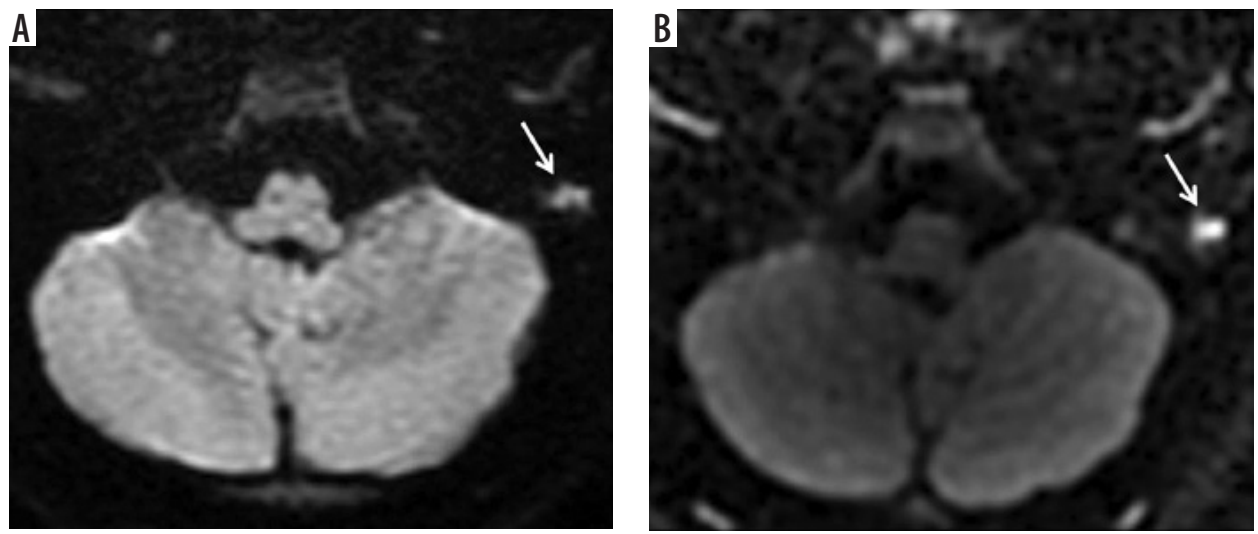

Figure 2. The example of worse diagnostic value of echo-planar imaging (A) compared to non-echo-planar (B) diffusion-weighted imaging in depicting middle ear cholesteatoma (arrows)
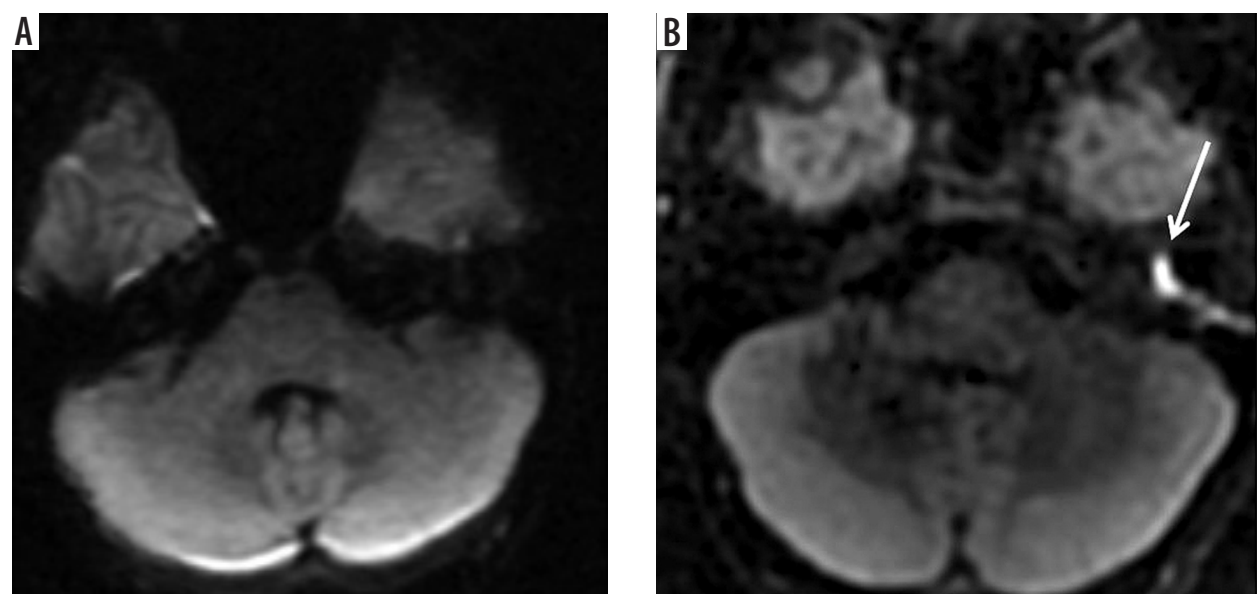

Figure 3. The example of no diagnostic value of echo-planar imaging (EPI) (A) compared to non-echo-planar (B) diffusion-weighted imaging (DWI) in depicting middle ear cholesteatoma (arrow). Cholesteatoma in not visible on the EPI DWI image (A) resulting in a false negative result
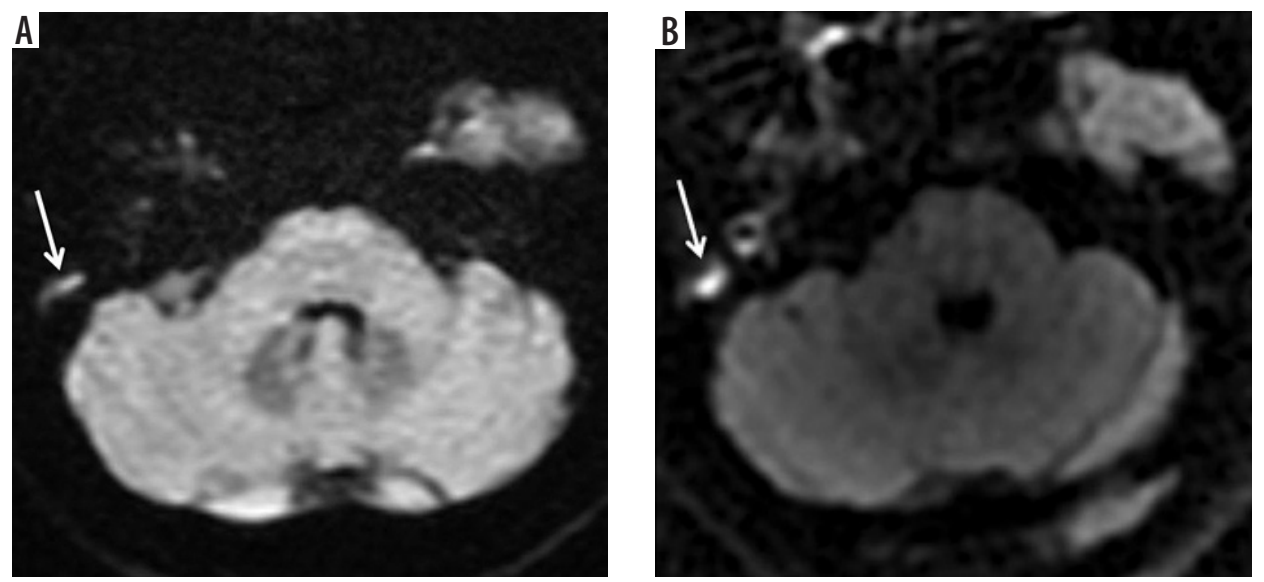

Figure 4. The example of restricted diffusion on both echo-planar (EPI) (A) and non-echo-planar (non-EPI) (B) diffusion-weighted imaging (DWI) interpreted as cholesteatoma. Postoperative histological result revealed only chronic otitis with no cholesteatoma - false positive result of both EPI and non-EPI DWI

planar DWI images [6-13]. The aim of the study was to compare the clinical usefulness and reliability of 2 different DWI techniques: EPI and non-EPI. EPI DWI is a fast technique routinely used in brain imaging, but there have been a few reports suggesting that it should be avoided in middle ear imaging. The aim of the study was to assess the clinical usefulness of this technique and to compare it with a non-EPI DWI performed on the same machine and evaluated by 2 independent readers experienced in the interpretation of DWI.

In our study the results of interpretation of EPI DWI showed poor correlation with histological findings. Based on EPI DWI, only $26.0-38.4 \%$ of cholesteatomas were correctly diagnosed, and $27-31 \%$ of cholesteatomas were 
diagnosed as chronic otitis without cholesteatoma leading to false negative results. The large number of false negative results make EPI DWI an unreliable technique that is not useful in clinical practice. Misdiagnosed cholesteatomas ranged in size from 4 to $25 \mathrm{~mm}$. It must be stressed that when using EPI DWI even a large cholesteatoma $(25 \mathrm{~mm}$ in size) was not diagnosed by both readers.

On the other hand, non-EPI DWI showed very high correlation with histological results, which is in concordance with the literature [11,15,17-19]. All cholesteatomas were correctly diagnosed by both readers, only 1 case of chronic otitis was overdiagnosed as cholesteatoma (false positive result). There were no false negative results using non-EPI DWI. Also, interobserver agreement in the evaluation of non-EPI DWI was very high, reaching $100 \%$. These results are in concordance with other reports and indicate that non-EPI DWI is a very reliable technique in detecting cholesteatoma [1,8,13-15]. As suggested in the literature, it may be used in the detection of residual as well as recurrent cholesteatoma, which is very important in treatment planning and may replace a second-look surgery of the middle ear [1,8,13-15]. It must be stressed that in our study both techniques, EPI and non-EPI DWI, overdiagnosed 1 case of chronic otitis as otitis with cholesteatoma. False positive results may lead to unnecessary ear operations while false negative results may cause postponing of the surgery and further middle ear destruction by a cholesteatoma $[6-8,14]$.

In our study there was a very low accordance between the results of EPI DWI and non-EPI DWI for each reader, indicated by poor intraobserver agreements. Each reader gave the same scores using EPI and non-EPI in less than $50 \%$ of the cases. Moreover, the assessment of EPI DWI showed also very poor agreement between the 2 readers in both pre- and postsurgical cases, which shows that evaluation of EPI DWI by different radiologists brings different results, which is not acceptable in clinical practice $[1,3,13]$.

Compared to non-EPI DWI, EPI DWI revealed much lower values of sensitivity and specificity as well as NPV and PPV, which is in agreement with previous reports [9-15,17-19]. In our research the sensitivity and specificity of EPI DWI were estimated as $69.2 \%$ and $66.6-83.3 \%$ for both readers, respectively. In the literature it has been reported that the EPI DWI technique may show higher sensitivity, reaching as much as $80 \%$, but only in cases of lesions larger than $5 \mathrm{~mm}$, but its sensitivity tends to drop to as little as $12.5 \%$ in lesions smaller that $4 \mathrm{~mm}$ [6-13]. In our study even cholesteatomas larger than $5 \mathrm{~mm}$ were misdiagnosed by both readers using EPI DWI. The main reason for such a weak performance of EPI DWI is its high sensitivity to susceptibility artifacts caused by the close vicinity of bone structures that obscure the middle ear pathology and may cause false positive or false negative results $[1,8,12]$.

Our research revealed very high values of sensitivity and specificity of non-EPI DWI in detecting cholesteato- ma, reaching $100 \%$ and $83.3 \%$, respectively, as well as high NP and PP values, reaching $100 \%$ and $96.3 \%$, respectively. A meta-analysis by Lingam et al., based on 26 studies, found non-EPI DWI to reach a sensitivity and specificity of $91 \%$ and $92 \%$, respectively [18]. The reason for such good results of non-EPI DWI sequences is that they use halfFourier single-shot-turbo spin-echo acquisition (HASTE) or multi shot turbo spin-echo acquisition with central kspace oversampling (PROPELLER, BLADE), which minimize motion and susceptibility artifacts, and geometric distortions, and increase the signal-to-noise ratio and spatial resolution with slice thickness less than $4 \mathrm{~mm}$ [5-15]. Moreover, Lips et al. retrospectively evaluated 135 patients with non-EPI DWI using $1.5 \mathrm{~T}$ and $3 \mathrm{~T}$ MR scanners and observed significantly lower sensitivity and specificity for $3 \mathrm{~T}$ in comparison to $1.5 \mathrm{~T}$ due to increased susceptibility artifacts at higher field strength. Thus, the researchers recommend imaging of cholesteatoma with 1.5 T MR scanners [20]. The patients in our study were examined using nonEPI PROPELLER DWI with a section thickness of $4 \mathrm{~mm}$ using a 1.5 T MR scanner, and this method was found to be very reliable and conclusive almost in every evaluated case apart from 1 misdiagnosed case.

In our study we did not evaluate the values of the apparent diffusion coefficient (ADC) derived from DWI. In the literature there are contradictory reports on the importance of ADC map analysis and measurements [11,15,21-25]. Magnetic field inhomogeneity, size of the lesion $(<3 \mathrm{~mm})$, utility of different MR machines of different vendors, and variable field strengths are the main limitations to obtaining a standardized cut-off value of $\mathrm{ADC}$ for cholesteatoma versus other pathologies in the temporal bone. Dremmen et al. and Schwartz et al. did not find ADC maps helpful as a method $[12,15]$. On the other hand, Özgen et al. emphasised the importance of ADC values and SI ratio measurements, suggesting that quantification parameters might reduce false positive results of non-EPI DWI [23]. Razek suggests that ADC measurements might help in differentiating malignant from benign lesions in the external ear [25].

\section{Conclusions}

Compared to EPI DWI, non-EPI DWI is a very reliable MRI technique for the diagnosis of cholesteatoma in the pre- or postoperative middle ear, with very high sensitivity and specificity as well as very high interobserver agreement, which make this technique very useful in clinical practice. EPI DWI, which is a standard sequence in brain imaging, is not recommended for the diagnosis of the middle ear cholesteatoma due to its low sensitivity and reproducibility between readers.

\section{Conflict of interest}

The authors report no conflict of interest. 


\section{References}

1. Baráth K, Huber AM, Stämpfli P, et al. Neuroradiology of cholesteatomas. Am J Neuroradiol 2011; 32: 221-229.

2. Mostafa BE, El Fiky L. Congenital cholesteatoma: the silent pathology. ORL J Otorhinolaryngol Relat Spec 2018; 80: 108-116.

3. Bernardeschi D, Russo FY, Nguyen Y, et al. Management of epi- and mesotympanic cholesteatomas by one-stage trans-canal atticotomy in adults. Eur Arch Otorhinolaryngol 2016; 273: 2941-2946.

4. Gamaleldin OA, Elsebaie NA, Khalifa MH, et al. Assessment of mass effect sign at high-resolution computed tomography in prediction of cholesteatoma. J Comput Assist Tomogr 2019; 43: 288-293.

5. Henninger B, Kremser C. Diffusion weighted imaging for the detection and evaluation of cholesteatoma. World J Radiol 2017; 9: 217-222.

6. Yiğiter AC, Pınar E, İmre A, et al. Value of echo-planar diffusionweighted magnetic resonance imaging for detecting tympanomastoid cholesteatoma. J Int Adv Otol 2015; 11: 53-57.

7. Dubrulle F, Souillard R, Chechin D, et al. Diffusion-weighted MR imaging sequence in the detection of postoperative recurrent cholesteatoma. Radiology 2006; 238: 604-610.

8. Vercruysse JP, De Foer B, Pouillon M, et al. The value of diffusionweighted MR imaging in the diagnosis of primary acquired and residual cholesteatoma: a surgical verified study of 100 patients. Eur Radiol 2006; 16: 1461-1467.

9. De Foer B, Vercruysse JP, Bernaerts A, et al. Middle ear cholesteatoma: non-echo-planar diffusion-weighted MR imaging versus delayed gadolinium-enhanced T1-weighted MR imaging - value in detection. Radiology 2010; 255: 866-872.

10. De Foer B, Vercruysse JP, Bernaerts A, et al. The value of single-shot turbo spin-echo diffusion-weighted MR imaging in the detection of middle ear cholesteatoma. Neuroradiology 2007; 49: 841-848.

11. Más-Estellés F, Mateos-Fernandez M, Carrascosa-Bisquert B, et al. Contemporary non-echo planar diffusion-weighted imaging of middle ear cholesteatomas. Radiographics 2012; 32: 1197-1213.

12. Schwartz KM, Lane JI, Bolster BD, et al. The utility of diffusion weighted imaging for cholesteatoma evaluation. Am J Neuroradiol 2011; 32: 430-436

13. Steens S, Venderink W, Kunst D, et al. Repeated postoperative follow-up diffusion-weighted magnetic resonance imaging to detect residual or recurrent cholesteatoma. Otol Neurotol 2016; 37: 356-361.
14. Van Egmond L, Stegeman I, Grolman I, et al. A systematic review of non-echo planar diffusion-weighted magnetic resonance imaging for detection of primary and postoperative cholesteatoma. Otolaryngol Head Neck Surg 2016; 154: 233-240.

15. Dremmen MHG, Hofman PAM, Hof JR, et al. The diagnostic accuracy of non-echo-planar diffusion-weighted imaging in the detection of residual and/or recurrent cholesteatoma of the temporal bone. Am J Neuroradiol 2012; 33: 439-444.

16. Razek AA, Ghonim MR, Ashraf B. Computed tomography staging of middle ear cholesteatoma. Pol J Radiol 2015; 80: 328-333.

17. Dhepnorrarat RC, Wood B, Rajan GP. Postoperative non-echo-planar diffusion-weighted magnetic resonance imaging changes after cholesteatoma surgery: implications for cholesteatoma screening. Otol Neurotol 2008; 30: 54-58

18. Lingam RK, Bassett P. A meta-analysis on the diagnostic performance of non-echoplanar diffusion-weighted imaging in detecting middle ear cholesteatoma: 10 years on. Otol Neurotol 2017; 38: 521-528.

19. She NN, Zhang XB. Diagnostic value of magnetic resonance imaging for middle ear cholesteatoma: a meta-analysis. Lin Chung Er Bi Yan Hou Tou Jing Wai Ke Za Zhi 2017; 31: 1833-1838 [Article in Chinese]. 20. Lips LM, Nelemans PJ, Theunissen FMD, et al. The diagnostic accuracy of $1.5 \mathrm{~T}$ versus $3 \mathrm{~T}$ non-echo-planar diffusion-weighted imaging in the detection of residual or recurrent cholesteatoma in the middle ear and mastoid. J Neuroradiol 2020; 47: 433-440.

21. Osman NMM, Rahman AA, Ali MTAH. The accuracy and sensitivity of diffusion-weighted magnetic resonance imaging with Apparent Diffusion Coefficients in diagnosis of recurrent cholesteatoma. Eur J Radiol Open 2017; 4: 27-39.

22. Lingam RK, Khatri P, Hughes J, et al. Apparent diffusion coefficients for detection of postoperative middle ear cholesteatoma on non-echoplanar diffusion-weighted images. Radiology 2013; 269: 504-510.

23. Özgen B, Bulut E, Dolgun A, et al. Accuracy of turbo spin-echo diffusion-weighted imaging signal intensity measurements for the diagnosis of cholesteatoma. Diagn Interv Radiol 2017; 23: 300-306.

24. Russo C, Elefante A, Di Lullo AM, at al. ADC benchmark range for correct diagnosis of primary and recurrent middle ear cholesteatoma. Biomed Res Int 2018; 24: 7945482.

25. Razek AAKA. Assessment of masses of the external ear with diffusion-weighted MR imaging. Otol Neurotol 2018; 39: 227-231. 